\author{
Do Paramedics Need to Learn Cultural Understanding \\ to Manage Acute Health Events Effectively? \\ C. Spencer, ${ }^{1}$ F. Archer ${ }^{2}$ \\ 1. Monash University Centre for Ambulance and Paramedic \\ Studies, Australia \\ 2. Monash University, Victoria, Australia
}

Globalization and migration are common reasons to justify the need for an awareness of cultural differences in healthcare situations. The belief is that understanding a patient's cultural background will achieve improved health outcomes. Such optimism and idealism are admirable goals to aim for in a non-emergency event, but in an acute health event paramedics have a limited time frame to treat patients, let alone get to know the patients' cultural backgrounds. The literature provides some generic guidance, but the specific literature about the cultural context of paramedic care is lacking.

This presentation explores different models for crosscultural health care and critiques their usefulness for paramedics working in the prehospital emergency health event.

Anecdotal evidence suggests that cultural factors do affect paramedic emergency care adversely. The initial stages of a qualitative research project also are presented as a first step toward systematically gathering evidence on this important topic. Finally, the paper demonstrates the critical need for understanding cultural awareness and that for paramedics there is an additional level of complexity in preparing them to recognize inconspicuous factors in an acute health event. Instead of classifying cultural issues as being too difficult to work on, this paper proposes a culturally responsive approach in the emergency setting and suggests a method for achieving such an outcome.

Keywords: acute; carc; cross-cultural; culture; cvents; health; manage; paramedics; understanding

Prehosp Disast Med 2005;20(2)::47

Qualitative and Quantitative Assessment of an Emergency Medicine Training Course in the Palestinian Territories

R. Retezar, ${ }^{1}$ G. Greenough ${ }^{2}$

1. Johns Hopkins Department of Emergency Medicine, Baltimore, Maryland USA

2. Center for International Emergency, Disaster and Refugee Studies (CIEDRS), Johns Hopkins Bloomberg School of Public Health, Baltimore, Maryland USA

Most international training courses are evaluated immediately after the end of the course using questionnaires and/or using pre- and post-test scores. Rarely is there an evaluation conducted to determine how these courses affect participants' clinical practice. Such an analysis was undertaken using by-person factor analysis to determine the impact of a training course in the Palestinian Territories. The Johns Hopkins Center for International Emergency, Disaster, and Refugee Studies (CIEDRS), in collaboration with the Cooperative for Assistance and Relief Everywhere, Inc (CARE), a private humanitarian organization, introduced the Emergency Medical Education and Development (EMED) course in the Palestinian Territories.
This six-day training program for physicians and nurses was established in collaboration with the Palestinian Ministry of Health and was designed to serve as a model for establishing the best practices in medical and trauma care. Initially, the program involved the training of 56 trainers, who subsequently went on to train an additional 528 physicians and nurses from tertiary, secondary, and, level-four, primary healthcare facilities. Six months after its implementation, opinions and perceptions toward the EMED course were obtained from key informant interviews of emergency physicians and nurses, which generated twenty-three consensus statements. Providers who took the course (11 physicians, 15 nurses, and one emergency medical services (EMS) instructor) were asked to rank these statements in a defined, quasi-normal, distributiongrid based on their perceptions about the course-from those they most agreed with $(+3)$ to those they most disagreed with (-3). Factor analysis of the responses was then performed using PQMethod 10 software.

Overall, the course has had a positive impact on the way physicians and nurses provide care in emergency departments. Participants believe the course has given them better skills, more knowledge on prioritizing and managing critically ill patients, and an improved nurse-physician relationship. Also, participants believe they have gained a better understanding of the philosophy and principles of Emergency Medicine and Nursing. By grouping course participants' responses using the distribution-grid, distinctive opinions emerged as indicators of barriers to best course implementation and of future educational courses.

By-person factor analysis, or Q-methodology, combines qualitative and quantitative data and, as such, can be a useful tool for evaluating international emergency medicine courses and their applications. Applied to EMED, this method has demonstrated that the course has improved the way participants deliver care.

Keywords: assessment; course; medical; Palestine; qualitative; quantitative; territories; training

Prehosp Disast Med 2005;20(2):547

\section{The Usefulness of Hypothetical Exercises in Disaster Preparedness \\ H. Harley}

Edith Cowan University and Royal Perth Hospital, Australia

Introduction: Hypothetical exercises are widely considered to be an appropriate method for evaluating the practicalities of emergency and disaster plans, and also to train staff in working in emergency, disaster situations.

Methods: A study was conducted at Royal Perth Hospital to assess whether the hypothetical exercises conducted in the year preceding the Bali bombings had adequately evaluated the Royal Perth Hospital Disaster Plans. The study also assessed whether the hypothetical exercises provided the necessary staff training to assist the staff in their responses to the bombings in Bali. The staff was randomly selected from a stuffy population of staff who were believed to have been involved in the hospital's response to the Bali bombings. 
Results: Only $26 \%$ of the staff that returned the questionnaire actually had participated in any of the hypothetical exercises. Therefore, the exercises provided only limited training to a small number of the staff involved in the response. The staff involved in the Bali response believed that their years of experience and knowledge of Royal Perth Hospital were what assisted them most in their response. Moreover, the results indicated that only $22 \%$ of the staff was aware of changes to the disaster plan postexercise.

Conclusions: However, in the Royal Perth Management Report (2002), ${ }^{1}$ which was written on the "Bali Bombing Incident", two recommendations regarding changes to the plan were made, therefore indicating that the previous exercises had not evaluated adequately parts of the disaster plan, particularly in relation to the care of relatives.

\section{Reference}

1. Harley H: Bali Bombing Incident. Royal Perth Hospital Management Report. Unpublished Report. Perth, Western Australia, 2002.

Keywords: Australia; disaster planning; hypothetical exercises Prebosp Disast Med 2005;20(2):s47-a48

Knowledge, Attitudes, and Behavior of Occupational Physicians Related to Burn Cases: A Cross-Sectional Survey in Turkey

M. Haberal; A. Kut; I. Tokalak; O. Basaran; G. Moray

Baskent University Faculty of Medicine, Turkey

Introduction: Occupational physicians provide the primary care of victims of burn cases at the workplace. In Turkey, it is routine to certify physicians for work in occupational medicine. This study aimed to evaluate the knowledge, attitudes, and behavior of occupational physicians regarding burn cases.

Methods: A total of 510 occupational physicians working in Ankara, Turkey were surveyed by mail, and $101(19.8 \%)$ physicians responded. Most of these physicians ( $\mathrm{n}=67$, $66.3 \%$ ) had encountered burn cases within the previous year. Results: The most frequent type of burn injury was scalds $(\mathrm{n}=55,54.5 \%)$ followed by thermal injuries $(\mathrm{n}=37$, $36.6 \%)$. Of the respondents, $22(21.8 \%)$ knew the most appropriate classification of burn injuries. Regarding firstaid treatments prior to triage, only $4 \%$ chose the valid items. The mean rate of positive attitudes of the participants toward the first aid of different types of burn injuries was $70.8 \%$. Only $31.7 \%$ of the physicians surveyed used up-to-date, first-aid modalities.

Conclusion: Turkish occupational physicians have inadequate knowledge and inappropriate attitudes toward the first aid and primary care of burn victims. With the vital support of the Burn and Fire Disaster Institute at Baskent University, nationwide educational policies could be improved and assessed.

Keywords: attitudes; behavior; burn; first aid; occupational; physicians; primary care; Turkey

Prehosp Disast Med 2005;20(2): 548

\section{Free Papers Theme 16: Public Health-2}

\section{Free Papers Theme 17:Tsunami-2}

\section{Theme 12: Emergency Medical Services Systems Design \\ Chair: Frank Archer}

\author{
Effective Planning for Disasters: The Hospital \\ Response \\ M.C. Trotta; C. Barletta; M. Gregori; P. Barletta; \\ M. De Simone
}

Emergency Department, Sant'Eugenio Hospital, Italy

During a natural or human-made disaster, the hospital emergency physician cannot flee; the physician must receive the casualties and decide the right course of action, based on the gravity and number of victims. The physician must plan the response of all the hospital services and key figures (anesthetist, surgeon, traumatologist, vascular and or thoracic surgeon, chief of nurses, chief of security). It fundamentally is important that the emergency department follow an "all-hazards" approach to achieve a competent and efficient hospital response regardless of the situation (natural disasters, such as earthquakes or storms, or human-made disasters, such as chemical, nuclear, or biological attacks).

Analyses of recent mass-casualty incidents (Tokyo 1995, New York 2001, Madrid 2004) have confirmed that $80 \%$ of casualties make their way to the nearest hospital on their own. Because they are not triaged on-site, there is a risk that the hospital could be overwhelmed by casualties, causing it to become ineffective. Protecting the hospital resources is a high priority in such situations, and the emergency physician's role is crucial. The authors describe the methodology for building an effective plan to handle a massive influx of casualties. Six essential stages will be highlighted: (1) education; (2) risk analysis; (3) inventory of resources; (4) possible scenarios; (5) management; and (6) action.

Keywords: disaster; emergency department; hospital; Italy; planning Prehosp Disast Med 2005;20(2)::48

\section{Preparing a National Health Emergency Plan S. Brazier \\ Ministry of Health, New Zealand}

Introduction: Following the shocks of 11 September 2001 and the severe acute respiratory syndrome (SARS), the New Zealand Ministry of Health stepped up preparations and is now completing a National Health Emergency Plan (NHEP) that encompasses the whole health sector.

Background: Like all countries, New Zealand faces the risks of terrorism and pandemics. In an increasingly complex and unsafe world, it is clear that central health agencies must take a key role in focusing on public health threats.

The New Zealand health sector has a devolved model with central funding and local autonomy. However, any response to national health emergencies must necessarily involve central planning and national control. The National Health Emergency Plan has dealt with these tensions by developing a modular and free form plan from which local health managers can select the elements they 